\title{
Palliative surgery and the surgeon's role in the palliative care team: a review
}

\author{
Anna D. Louie, Thomas J. Miner \\ Department of Surgery, Brown University, Lifespan Health System and Warren Alpert Medical School, Providence, RI, USA \\ Contributions: (I) Conception and design: Both authors; (II) Administrative support: Both authors; (III) Provision of study materials or patients: None; \\ (IV) Collection and assembly of data: None; (V) Data analysis and interpretation: Both authors; (VI) Manuscript writing: Both authors; (VII) Final \\ approval of manuscript: Both authors. \\ Correspondence to: Thomas J. Miner, MD, FACS. Professor of Surgery, The Warren Alpert Medical School of Brown University, Providence, RI, Chief \\ of Surgical Oncology, Rhode Island Hospital, 593 Eddy Street - APC 439, Providence, RI 02903, USA. Email: thomas.miner@brownphysicians.org.
}

\begin{abstract}
This review focuses on the role of palliative surgery in the care of the palliative care patient, and the appropriate role of the surgeon. The surgeon has much to bring to the palliative care team. The surgeon's role goes beyond the technical requirements of the palliative procedure, which itself must be strictly defined, and has recognized utility for improving quality of life in selected patients. These benefits may be substantial, but come at significant risk; requiring careful balancing of risks and benefits that is most completely understood by the surgeon. The surgeon's judgement can help determine which procedure best meets a patient's goals. The complex dialogue involved in the decision to undergo a palliative operation requires excellent communication between the palliative care team, the patient, and their family. Integrating the surgeon into the palliative care team could help with earlier initiation of those palliative discussions, and assist deliberation of palliative surgery. Surgeons also understand the importance of communication around palliative surgical interventions and have adapted several teaching models to the specifics of this crucial communication. A palliative team combining both surgeons and palliative care physicians may promote goalconcordant decision-making and remove barriers to communication and team engagement. The future of palliative surgery research will involve measures of success that go beyond physiology or mortality, to include more evaluations of communication and patient goals.
\end{abstract}

Keywords: Palliative surgery; communication; risk-benefit analysis; palliative care team

Submitted Dec 01, 2020. Accepted for publication May 26, 2021.

doi: 10.21037/apm-20-2381

View this article at: http://dx.doi.org/10.21037/apm-20-2381

\section{Introduction}

In a world where surgery can improve overall survival even in patients with unresectable metastatic cancers (1-4), palliative surgery is, in part, defined by what it is not. An operation would not meet the definition of palliative surgery if the goals for surgery include alteration of overall disease progression or patient survival. This would hold true even for operations performed on patients receiving palliative care or done without curative intent. Indeed, one fifth of Medicare beneficiaries undergo an operation in their final month (5). Without an understanding of the surgical goals, these procedures should not be considered palliative surgery. In palliative surgery, symptom relief and quality of life must be the primary focus of the surgeon and the palliative team. Only a subset of noncurative cancer resections or operations performed within the last months of life will meet this definition. Palliative goals should be explicitly acknowledged in the preoperative documentation (6), particularly when creating inclusion criteria for retrospective research. Palliative surgery can be defined as an operation that is "largely intended for symptom relief or avoidance of symptoms or conditions anticipated secondary to progressive local disease, and is unlikely to alter the ultimate progression of disease in 
this patient or significantly impact patient survival." (7). The effectiveness of palliative surgery is based on the durability of patient-acknowledged symptom resolution (8).

Surgeons are familiar with palliative goals and have long operated with such goals in mind. Many operations, such as the original Halstead radical mastectomy and coronary artery bypass grafting, were first advocated for symptom relief and pain management, and only later found to improve survival $(9,10)$. Palliation is still integral to surgical practice, and the American Board of Surgery specifically mentions palliation in defining the specialty of general surgery (11). Indeed, it was a surgeon, Dr. Balfour Mount, who coined the term "palliative care" (12). Surgeons have repeatedly shown their commitment to palliation. In 2012, at the behest of the American College of Surgeons, the Cancer Program Standards developed by the Commission on Cancer for program accreditation required that palliative care services be available to patients (13). The American College of Surgeons has also revised the standard surgical morbidity and mortality outcome measures to accommodate palliative surgery (13).

This ongoing surgical commitment to palliation is also reflected in the frequency of palliative surgery. At one major cancer center, $40 \%$ of all surgical consultations are for palliative surgical consideration, and of those palliative consults, $43 \%$ ultimately receive an operation and $17 \%$ receive a procedural intervention (12). At major US cancer centers, $13 \%$ of all operations are palliative (12). Some palliative procedures can even be performed on an outpatient basis, making up $21 \%$ of all palliative interventions at one comprehensive cancer center (14). Certain surgical subspecialties have even higher rates of palliative surgery. Vascular surgeons often prioritize symptom control and quality of life in their approach to conditions like peripheral vascular disease and may view these procedures as palliative interventions (15). In thoracic surgery, as much as $23 \%$ of all procedures performed are palliative (16). Within comprehensive cancer centers, palliative procedures make up a significant minority of all cases performed by other surgical subspecialties, such as neurosurgery $(46 \%)$ and orthopedic surgery (31\%) (14). While these cases likely cluster at specialized cancer centers, they reveal surgeons' willingness and commitment to improving patient quality of life through palliative surgery.

Some common indications for palliative surgery are malignant bowel and biliary obstruction, management of extensive fungating skin and soft tissue disease, peripheral arterial disease, pleural and pericardial effusions, tracheobronchial obstructions, hemoptysis and bronchopleural fistula (16). These conditions can cause debilitating symptoms that can be difficult to control. Examples of such symptoms include pain, nausea and inability to eat in malignant bowel obstruction; or jaundice, infection and malnutrition in biliary obstructions. Fungating breast cancers and locally advanced skin cancers, as well as bulky nodal disease, can cause painful malodorous wounds that bleed and require frequent care. Peripheral vascular disease can cause rest pain and non-healing wounds. Pleural and pericardial effusions can limit activity and exercise tolerance. All can be painful and negatively impact a patient's independence and quality of life.

\section{Risks and benefits of palliative surgery}

No surgical intervention can be considered without an analysis of the risks. This is particularly important in palliative surgery where symptoms, independence and need for hospitalization define the success of the procedure. Palliative surgery is higher risk than non-palliative operations when evaluated in terms of morbidity and mortality. The American College of Surgeons' National Surgical Quality Improvement Program data showed that any operation on a patient with disseminated malignancy has substantial morbidity and mortality rates. The 30 -day unadjusted morbidity and mortality rates of $28.3 \%$ and $8.9 \%$, respectively, were much higher than for the average surgical patient (17), indicating that these patients are particularly fragile. Even operations that are generally lowrisk can have substantial risks when applied to palliative patients. The overall mortality rate for percutaneous endoscopic gastrostomy (PEG) tube placement is $0.2 \%$ (18). Yet in a retrospective study of patients with malignancyin which $27 \%$ of PEG tube placements were for palliative decompression - the procedure was associated with $20 \%$ in-hospital mortality (19). The largest prospective study of palliative surgery outcomes, encompassing 1,022 patients, revealed an $11 \% 30$-day mortality (20). Similarly, a retrospective review of 884 palliative thoracic surgery procedures found $11 \%$ of patients died before leaving the hospital (16). While prolonging survival is not the main goal of palliative surgery, this significant risk of early postoperative mortality is relevant when discussing an individual patient's treatment preferences.

Palliative operations can come at the cost of many potential complications. The palliative team can draw on the surgeon's experience to understand potential complications, which will be highly dependent on patient 
and surgery-specific factors. For instance, major operative complications are endured by $30 \%$ of patients undergoing palliative operations for malignant bowel obstruction from gynecologic cancer (21). Any potential complications may increase a patient's hospital stay (12). Even malignant bowel obstruction patients who benefit from the operation with a resumption of diet may not be able to avoid periods of hospitalization afterwards (11-61\% of remaining lifespan) (22). Recovering from palliative operations sometimes demands physical resources that outstrip the reserves of an already-ill patient. In a small prospective study that tracked complications in great detail, even some of the most subjectively robust patients suffered from adverse events (20).

While the surgeon may not always be able to predict and prevent complications, their familiarity with the specific procedure allows them to anticipate the nature and even potential frequency of complications. In one study of palliative gastrectomies, the morbidities were similar to those of other noncurative gastrectomies (6). The similarity suggests that surgeons can extrapolate from other advanced cancer cases, and contribute helpfully to discussions of palliative surgery risks and benefits. Experienced surgeons have a deep understanding of the high-risk nature of palliative surgery and will carefully weigh these risks when suggesting treatment options to the patient and the palliative team.

Despite the drawbacks, palliative surgery can improve patients' symptoms and quality of life, and should be considered for certain patients in discussion with the palliative surgeon. In carefully selected patients, the benefits of palliative surgery can be significant. In a study of 1,022 patients undergoing palliative procedures, patients had a median survival of 194 days, and a quarter of patients survived over 350 days after the operation (23). This shows that many patients survive long enough to see substantial benefits from palliative surgery. A large retrospective study of palliative thoracic procedures similarly had a median survival of 110 days and most patients were able to discharge home (16).

Palliative surgery can result in substantial, lasting improvement in symptoms for the majority of patients. A large prospective study of palliative oncologic surgery found that $80 \%$ of patients had symptom improvement or resolution within 30 days, with a median of 135 days of symptom control (23). In a retrospective review of 193 patients whose malignant bowel obstruction was treated surgically, 91\% were able to consume a solid diet for more than two weeks and be discharged home from the hospital (24). Similarly, in a
2000 Cochrane review of palliative operations for malignant bowel obstruction surgical treatment was associated with postoperative control of symptoms in $42-80 \%$ of patients. However, the included studies had varying reobstruction rates (10-80\%) and little detail on time to reobstruction (25). Another study saw relief of obstruction and improvement of symptoms in $40-70 \%$ of patients after surgery (26). These significant improvements in symptoms are found in other types of palliative surgery, including major procedures such as a palliative gastrectomy, which provided successful symptom control without need for further palliative interventions in $76 \%$ of patients (6). When surgery resolves symptoms, it usually does so within 30 days (27). A larger study of 227 patients confirmed the rapid resolution of symptoms after surgical intervention, and found resolution or improvement of symptoms in $90.7 \%$ of patients (28). A small study including a wide variety of palliative surgical procedures revealed that the symptom improvement was sustained for a median of 3.4 months postoperatively (20). The speed and durability with which symptom resolution can be achieved surgically is important in patients with limited life expectancy.

Studies of palliative surgery have also directly addressed quality of life and found improvements across a variety of surgical interventions. A study of palliative spinal surgery showed improved quality of life in patients undergoing surgery as the operation relieved pain and reduced reliance on medications (29). A review of surgery for malignant bowel obstruction concluded that if carefully selected, a majority of patients who undergo palliative surgery can achieve quality of life improvements (21). In a comparison of palliative surgical and endoscopic interventions for malignant gastric outlet obstruction, at 30 and 60 days the surgical group had significantly better oral intake. After adjusting for survival, surgery was also associated with more total days tolerating oral intake (30). This study recommended surgery for patients with a life expectancy of at least 2 months, as it provided better quality of life in those patients. Many of the most challenging symptoms of palliative patients can be addressed surgically, improving quality of life.

For some patients, deliberately addressing life-limiting symptoms surgically may allow more goal-concordant decision making and therefore improve the patients' physical, emotional and social well-being, and quality of life (31). While surgery is often assumed to come at a cost to quality of life, at least during the immediate recovery, a study of palliative surgery patients showed no diminution in quality of life postoperatively, even in those who did not 
experience tangible benefits (27). In fact, the significant improvements in physical and functional well-being postoperatively are present even in a study where $54 \%$ of patients did not achieve the desired operative result (18). The best judge of the value of palliative surgery may be the patients themselves. In two studies encompassing a wide variety of palliative operations, 88 and $90 \%$ of patients felt that the operations were "worth it", even though some experienced complications. Therefore, the significant risk of complications that is a potential downside of surgery may be less relevant in palliative patients since it does not appear to decrease their quality of life. Perhaps these patients find the active pursuit of symptom resolution psychologically valuable even when it is not fully achieved.

\section{Surgical judgement in palliative procedures}

Palliative procedures encompass a wide range of interventions, from comparatively minor endoscopic and surgical interventions to complex and invasive operations. Surgeons are intimately familiar with the magnitude and recovery time of various interventions, and that insight is highly valuable when determining which interventions are consistent with a patient's goals. For instance, with a highly selective and individualized approach, even a pelvic exenteration, which conveys an exceedingly high morbidity, can provide symptom relief in $79 \%$ of patients (32). However, the 3-6 months recovery time associated with a procedure as extensive as pelvic exenteration can make it difficult to achieve quality of life improvements in a palliative patient (21). While this is an extreme example, the surgeon's familiarity with the potential costs and benefits of both surgical interventions and endoscopic procedures makes their judgement an asset to the palliative care team.

Surgery and minimally invasive or endoscopic procedures can be seen as complimentary tools for meeting the needs of palliative patients. In certain situations, endoscopic procedures can be extremely successful. Palliative transurethral prostate resection, for instance, has a lower morbidity and a $70 \%$ success rate without requiring the urinary diversion common to more extensive urinary tract operations (33). For malignant bowel obstruction, both surgical and endoscopic procedures, when feasible, allowed resumption of eating, though only operation also restored bowel function (18). Perhaps because endoscopic procedures are more frequently applied in those patients with shorter life expectancy, they do not show mortality benefits over surgical interventions $(12,23)$. When short term success and avoidance of complications is the highest priority, endoscopic procedures may be appropriate. For instance, Moss et al. found that when treating obstructive jaundice from pancreatic cancer, stents were associated with decreased risk of complications and no difference in shortterm technical success rates (34). The reduced risk of shortterm complications with endoscopic procedures makes them a valuable tool in the palliative arsenal. However, there was a substantial difference in the rate of reintervention in the endoscopic vs. surgical group (36\% vs. 3\%), and more of the patients undergoing endoscopic procedures had biliary reobstruction prior to death (12). Gastrointestinal stents for gastric cancer were also associated with elevated rates of late complications compared to the operative group (44\% vs. $12 \%$ ) (35). While the short-term risk-benefit ratio may favor minimally invasive procedures, they do not have the durability of surgical interventions. For patients expected to survive somewhat longer, the benefits of surgery may outweigh those of endoscopic procedures.

Choosing a palliative intervention often requires practitioners to "walk a tightrope" between potential benefits and short-term harms. The surgeon can bring significant insight into the complex decision-making that comes with choosing any palliative procedure. Surgical operations are intrinsically painful, involve hospitalization, and at least in the short term a loss of autonomy (12). Patients may not fully understand what they are agreeing to in terms of recovery or complication risks when they request surgery (36), so it is important to involve a surgeon in the decision-making process. Even within the realm of palliative procedures, all of which should be considered higher risk, risk level varies widely between smaller procedural interventions, smaller operations, and major open operations. A more minimally invasive approach can also be applied to surgical interventions, such as favoring laparoscopic interventions over open abdominal surgery. In a study of surgeries for malignant bowel obstruction, laparoscopic operations had a decreased rate of surgical site infection (4\% vs. 32\%), with no significant difference in ability to tolerate solid food (24). A surgeon will be able to provide insight into which interventions are feasible in a particular patient, and most concordant with their goals of care given the balance of risk and benefit.

\section{Preoperative decision-making and palliative surgery}

Patient selection is crucial to good outcomes in palliative 
surgery (28), and thus the surgeon's judgement is a valuable addition to the palliative team's discussion and planning. For instance, in a patient with a malignant obstruction, some forms of obstruction, such as a single-site obstruction, are more amenable to surgical intervention, whereas multisite obstructions or those with ascites and poor functional status do not respond well to surgical intervention (26). Because of the potential harms of surgery, palliative surgical procedures tend to be concentrated in more robust palliative patients. In patients undergoing a procedural intervention with palliative intent for pancreatic cancer, those undergoing surgical intervention had longer survival than those undergoing other procedures (37). This shows how seriously surgeons take the potential risks of palliative surgery and try to ensure that patients receive the procedure they can tolerate, and which will give them the best outcome.

Poor palliative surgery outcomes are associated with lower preoperative performance status, poor nutrition and weight loss (9). The surgeon's judgement takes into account these and many other patient factors, which may explain why in one palliative surgery series, median overall survival was four times longer in patients selected for palliative operations. This suggests that only those patients subjectively assessed to be most robust are considered appropriate for palliative surgery (28). Indeed, higher preoperative illness burden correlates with worse surgical outcomes (8), confirming the inherent importance of these surgical judgements. Surgeons will also understand the difficulty of achieving successful palliation through surgery. Surgical judgement about procedure durability indicates the achievability of symptom relief. In a prospective evaluation of palliative surgery, patients the surgeon predicted to have amelioration of the chief complaint for the remainder of their lives had a much longer duration of symptom improvement postoperatively (20). The surgeon knows what is possible, and that understanding of an individual patient's anatomy and operative options will contribute to the care discussion.

Bringing a surgeon into the palliative discussions early can help determine the timing and scope of a potential operation. Because a more physiologically robust patient has better palliative surgery outcomes, some surgeons have advocated preemptive palliative procedures for anticipated symptoms of disease progression. Preemptive palliative procedures for anticipated symptom occurrence have the same risks as those procedures in patients with active symptoms (9). While such a procedure can prevent anticipated symptoms and is associated with longer survival, that comes at a cost of notable upfront morbidity (28). This is a delicate tradeoff but may be warranted in selected situations when the palliative aims are clear. It is very important that the surgeon be fully aware of and in agreement with the patient's palliative goals of care preoperatively, as surgeons operate differently when they know the goal is palliation. Surgeons performing a gastrectomy operated differently when the goal was explicitly palliative, doing fewer high-risk esophageal anastomoses and taking fewer lymph nodes (6). The more a surgeon understands the patient's wishes, the more they can accommodate those wishes through procedure selection, timing and intraoperative decision-making.

Just as surgeons can and should tailor the procedural intervention to a patient's specific goals, they can shape the upfront discussion to help the patient decide what intervention best achieves their aims. High risk operations require complex decision-making (38), and the outcome may not always be clear even to the most experienced surgeon. However, surgeons can often agree on the overall prospects of seriously ill surgical patients. Even when there is no high-quality evidence to guide their decision-making, given a clinical vignette involving potential outcomes of surgery in a seriously ill older patient, $>85 \%$ of surgeons agreed in their prediction of the likely outcome (5). Additionally, some symptoms are more easily addressed surgically, allowing surgeons to give a patient a clearer picture of the achievability of their goals via surgery. For example, in rectal cancer resections, bleeding and obstruction were more often improved after operation, whereas effective pain control was less frequently obtained and less durable (9). When guiding a discussion of potential palliative interventions, "emphasis must be placed on those things that can be realistically delivered. It is reasonable for the patient with advanced cancer to hope for good quality of life, symptom resolution, technically superior palliative operations, dignity, and compassion." (9). The integral nature of surgical judgement to the discussion of palliative intervention shows how necessary the surgeon's insight is to the palliative care discussion.

\section{Improving palliative care discussions of surgical intervention}

Communication is an essential aspect of both palliative care and the planning of any surgical intervention. Productive discussions with the patient and family are crucial. Physician 


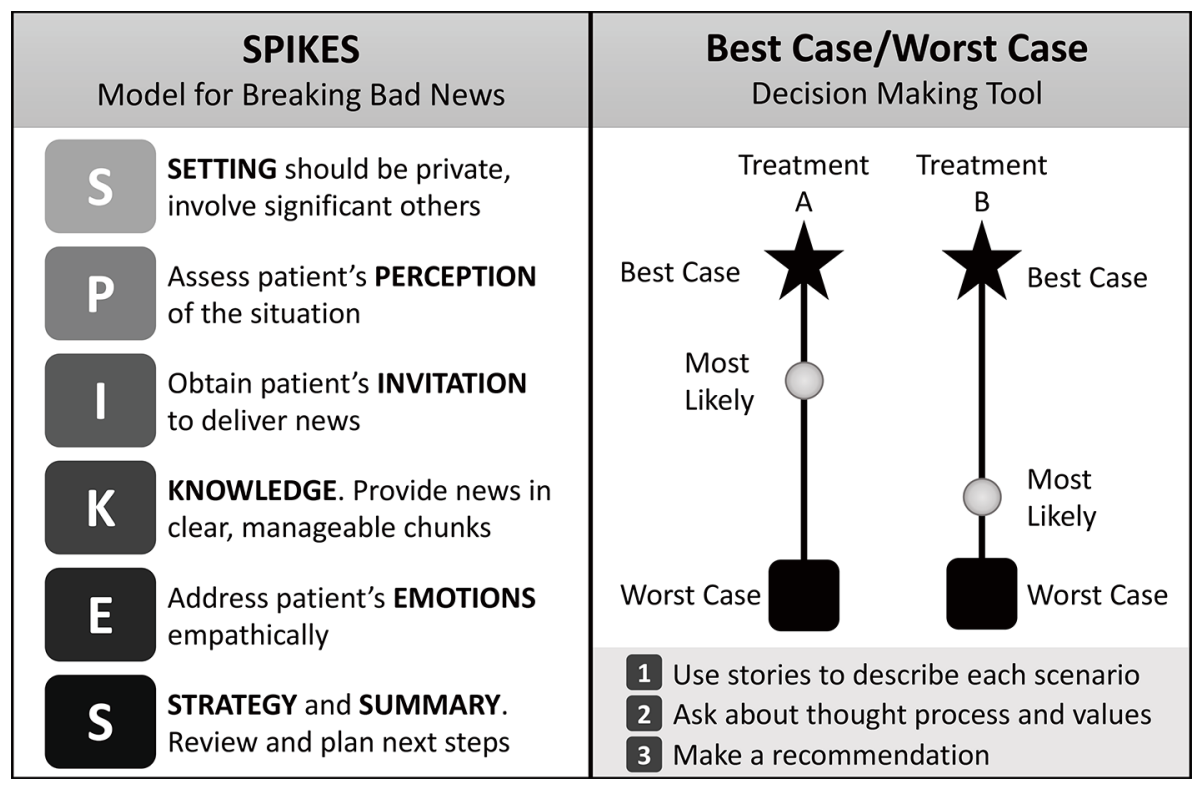

Figure 1 Depicts the steps of the SPIKES model for breaking bad news and the Best Case/Worst Case decision making tool. Adapted from work by Baile et al. (40) and Schwarze et al. (41).

communication skills are positively associated with patients' satisfaction, adjustment to illness and participation in care, and decreased surgeon malpractice liability (10). Goals of care discussions before a palliative procedure are associated with decreased ICU utilization, decreased readmissions and better quality of life (37). Surgeons understand the necessity of these conversations. Surgeons feel if they are contacted about potential interventions, it is their responsibility to discuss end of life with the patient, and not to offer futile interventions where the harm outweighs the potential benefit (5). Surgeons are often called upon to perform the heroics of medicine but feel great responsibility to avoid interventions without potential benefit. In support of this position, it has been proposed that with respect to seriously ill patients "doctors have a moral, ethical, legal responsibility to reduce pain and suffering but no moral, legal, or ethical obligation to interfere with the dying process." (26). Still, surgeons may struggle to preserve patient choice in the face of high-risk palliative surgery with uncertain outcomes (7). This is further complicated by the fact that patients often hold preferences for significant treatment limitations, but do not discuss them with the surgeon preoperatively (36).

Communication around palliative surgery may be particularly fraught, yet these skills are underemphasized in surgical training. Surgical residents are often in clinical scenarios where they are expected to discuss palliative care, and agree on the importance of this skill, but sometimes feel their training is inadequate (11). Surgical training rarely highlights explicit instruction on secondorder communication skills like conveying empathy and understanding. Many surgeons feel they should be better at communication than they are (10). There are few quality studies evaluating end-of-life communication training for surgeons (39) despite their frequent involvement in palliative care discussions. More research is needed to understand how commonly surgeons feel inadequately prepared for palliative care discussions. As palliative surgery and its associated discussions fall within the purview of the surgeon, surgical trainees could learn much from the training afforded to palliative care physicians. Surgical education should approach the shared-decision-making conversation as an instrument of care (8), and teach it with the rigor applied to other surgical skills.

Several models teaching improved communication have been applied to the preoperative discussion. Figure 1 depicts the SPIKES and Best Case/Worst Case communication models. The SPIKES communication model for delivering difficult news has been used to help surgeons improve the context and structure of their communications (10), but does not specifically address the complex decision-making of preoperative discussions. Even short interventions teaching the SPIKES communication model to surgical 
residents significantly increased the proportion of residents who felt comfortable discussing end-of-life issues, and $92 \%$ went on to use the information in clinical practice (11). Another communication model, Best Case/Worst Case (BC/ WC), is designed to help patients and families visualize the risks and benefits of different treatment options. When discussing $\mathrm{BC} / \mathrm{WC}$, patients and families found it clarifying and noted that it was helpful in their deliberation and in establishing expectations (42). Teaching surgeons how to use $\mathrm{BC} / \mathrm{WC}$ increases discussion of outcomes and treatment alternatives (43). Surgeons also feel BC/WC is practical and continue to use it in their clinical practice (42). Developed from a collaboration between surgeons and palliative care physicians, Best Case/Worst Case is an example of how many of the challenges around palliative surgery can be addressed by increasing the collaboration between surgery and palliative care teams.

\section{What surgeons can bring to the palliative care team}

Surgeons are a valuable addition to the palliative care team. Surgeons commonly have a long-term involvement with their patients. Palliative surgery patients have high rates of satisfaction with their care because the surgeon was there for them during a difficult time and remained engaged with them throughout the remainder of their lives (10). For patients who fear abandonment by their medical team, the presence of the surgeon on the palliative care team will add another provider dedicated to longterm physician-patient relationships. In comparing potential procedural interventions, the surgeon brings a wealth of knowledge to the discussion. A surgeon's risk/ benefit assessment is grounded in a robust fundamental knowledge of surgical outcomes and indications (44). This is important as palliative alternatives to surgery are often poorly regarded by patients, and they rarely remember the risks of interventions after discussion (7). Patients often feel that surgery is their best option and may be biased in their decision-making by the physical impact of their current symptoms outweighing future risks (45). This makes it imperative that the palliative team include a surgeon in their discussion so that any options presented to the patient are considered, individualized and achievable. After an informed discussion with a surgeon, $15.7 \%$ of patients considering palliative surgery felt the risks of complications were too high (18). The surgeon can bring clarity to the discussion, which benefits the patient. Targeted end-of- life discussions are known to improve quality of life for both patient and caregiver in part because they opt for less aggressive treatments when appropriate (46).

When it comes to decisions around palliative procedures, surgeons can not only determine when a procedure is appropriate, they can determine what procedures are most concordant with the patient's goals. Patients often see the decision to undergo an operation as a dichotomization between surgical life-prolongation and hospice (47). The surgeon can bring nuance to the discussion. Just as there is far more to a goals of care discussion than choosing to sign a Do-Not-Resuscitate order, the procedural conversation goes beyond a yes/no dichotomy. As discussed earlier, there is a range of potential palliative procedures available to patients, and the surgeon will understand which options best fit a particular patient. While they cannot fully predict the outcomes of a procedure, surgeons have an intuitive understanding of a patient's chances of a good surgical outcome, which can be measured by their response to the 'surprise' question (48). This knowledge will help the surgeon guide a nuanced discussion of which palliative procedures are most goal-concordant for the patient and family, which is another contribution they can bring to the early palliative care discussions.

\section{Advantages of a palliative care team integrating surgeons and palliative care physicians}

Surgeons also value the active involvement of the palliative care team. The decision-making required in palliative surgery can be an emotional process. One of the most common moral dilemmas that concerns surgeons is providing honest information without destroying hope (7). High-risk surgery may require the patient or family to make decisions for which they are unprepared, or to accept the possibility of a poor outcome (5). Surgeons may not always feel comfortable addressing these emotional reactions. Surgeons, too, develop a kind of surgical buy-in, where they feel a deep personal responsibility and guilt for any poor outcomes, and may assume that operative consent entails full agreement with aggressive postoperative care (44). Without a team approach, surgeons may perceive consultation as a loss of ownership over patient trajectory, and may feel that non-surgical teams lack insight into surgical risks and decision-making (44). This perception may be a barrier to palliative care involvement or cloud the surgeon's ability to respond to patient's wishes and fully address the patient's emotions. Palliative care physicians 
respond compassionately to expressions of emotional distress (49), which may help facilitate discussions between family, patient, palliative care team and surgical team.

A team approach to palliative surgical care has many advantages. Early involvement of the surgical team alongside palliative care promotes goal-concordant decision-making (31). The palliative triangle is an application of palliative care principles to surgical decision-making. Its approach is focused on nurturing a relationship between surgeon, patient and family to promote consistent communication and positive relationships. In a study of 227 patients, those selected for operative management using the palliative triangle had lower 30-day morbidity and mortality as well as superior symptom improvement. Indeed, $90.7 \%$ of those selected using the palliative triangle were found to have symptom improvement (18). This team dynamic need not necessarily be limited to the fields of surgery and palliative care. The team may occasionally need to include procedural specialists or anesthesiologists, such as when the intubation required for the operation will be difficult to reverse, and prolonged intubation is inconsistent with the patient's goals of care (50). Balancing the potential for complications with the possible benefits requires an understanding of the patient's expected lifespan. Physicians often have a difficult time recognizing when a patient is nearing the end of life (26). However, group discussion by a multidisciplinary team that included surgeons improved the group's accuracy in predicting survival in lung cancer patients (51), demonstrating that the interactions of the group make it stronger than the sum of its parts.

A team that can anticipate mortality can better anticipate which patients should fall under the purview of a combined palliative surgery team. Even in patients not undergoing explicitly palliative procedures, assessments of preoperative frailty that allowed for team discussions gave the team opportunities to modify perioperative plans. This improved outcomes and survival in frail patients (52). Similarly, there are palliative surgical procedures that should automatically trigger a multidisciplinary palliative team review. Some groups suggest that all surgical patients with carcinomatosis or unresectable malignancy should receive palliative team consultation (47). Surgeons are often consulted for palliative procedures in patients who have not received palliative consultation. At one institution, only $14 \%$ of thoracic patients who received palliative procedures had palliative care assessment before surgical consultation (16). Only an additional $5 \%$ of patients received a palliative care consultation during their stay, and it is unclear if those additional consultations occurred before or after the surgical procedure. A combined palliative surgery team may smooth the consultation process for medical teams considering palliative surgical interventions. Certain patients could also benefit from automatic consultations to a multidisciplinary palliative surgery team. For instance, at one institution, among patients receiving gastrostomy tubes, $90 \%$ died in the hospital or were severely disabled on discharge, but only $12 \%$ of those patients received a documented goals of care assessment prior to the procedure. Another institution with a $68 \%$ rate of documented goals of care discussions in patients undergoing gastrostomy tube placement, only $20 \%$ of those discussions occurred pre-procedure (53). Given the risks and discomfort involved in palliative surgical procedures, it is important that goals of care discussions precede any procedural intervention. Because gastrostomy tubes can be performed by many different services, a multidisciplinary palliative surgery team could better coordinate these goals of care discussions. There are likely other defined groups of patients who could benefit from standardized consultations to a multidisciplinary palliative surgery team.

\section{The future of palliative surgery}

There are still many barriers to good communication between the provider team, patients and their families. These communication barriers become particularly marked when patients are faced with difficult decisions such as the option to undergo a palliative operation. Surgeons cite several systemic barriers, such as time constraints, inadequate training, and lack of quality evidence (54). Teams that combine surgeons and palliative care physicians can better address some of the systemic barriers and gaps in consultations. Such teams could also promote improvements in palliative surgery research.

While the true goal of palliative surgery is quality of life and relief of symptoms, most articles do not focus on validated assessments of quality of life (9). In one review, a majority of studies focused on morbidity and mortality, with only $17 \%$ addressing quality of life, and only $40 \%$ of those using validated assessment tools (55). Even recent articles recommending palliative operations do so without assessing the impact on symptoms and continuing to place morbidity and mortality in the spotlight (56). Research is needed to identify better measures of success in palliative surgery instead of focusing primarily on physiology (45). New interdisciplinary research methods have been proposed to address this gap and have 
developed culturally sensitive consensus methodologies for future palliative surgery studies (57). However, much work remains to be done. Researchers should work to more widely implement palliative scoring systems. The McGill quality of life index, Quality of Life at End of Life (QUAL-E), and the Quality of Dying and Death Questionnaire (QODD) were the highest rated when scored for validity, internal consistency, interpretability, reliability and responsiveness (18). On a smaller scale, tracking simple patient-centered outcomes such as asking patients whether an intervention was "worth it" may allow for assessments of a procedure's value (18). Palliative surgery patients deserve a team that focuses on what is most important to them: their quality of life. Surgeons' traditional focus on morbidity and mortality is inappropriate in these patients. Using the classic setting of the morbidity and mortality conference to explore how successfully the operation met palliative goals may provoke the kind of cultural change that will allow for more contributions from surgeons to the palliative care team (26). The surgeon has much to add to the palliative care team. The surgeon's insights into the risks and benefits of palliative surgery can add clarity to perioperative palliative decision-making.

\section{Acknowledgments}

Funding: None.

\section{Footnote}

Provenance and Peer Review: This article was commissioned by the Guest Editors (Fabian M. Johnston and Ana Berlin) for the series "Palliative Care and Surgery" published in Annals of Palliative Medicine. The article has undergone external peer review.

Conflicts of Interest: Both authors have completed the ICMJE uniform disclosure form (available at https://apm. amegroups.com/article/view/10.21037/apm-20-2381/coif). The series "Palliative Care and Surgery" was commissioned by the editorial office without any funding or sponsorship. The authors have no other conflicts of interest to declare.

Ethical Statement: The authors are accountable for all aspects of the work in ensuring that questions related to the accuracy or integrity of any part of the work are appropriately investigated and resolved.
Open Access Statement: This is an Open Access article distributed in accordance with the Creative Commons Attribution-NonCommercial-NoDerivs 4.0 International License (CC BY-NC-ND 4.0), which permits the noncommercial replication and distribution of the article with the strict proviso that no changes or edits are made and the original work is properly cited (including links to both the formal publication through the relevant DOI and the license). See: https://creativecommons.org/licenses/by-nc-nd/4.0/.

\section{References}

1. Lau JW, Chang HS, Lee KY, et al. Survival outcomes following primary tumor resection for patients with incurable metastatic colorectal carcinoma: Experience from a single institution. J Dig Dis 2018;19:550-60.

2. Tarantino I, Warschkow R, Worni M, et al. Prognostic relevance of palliative primary tumor removal in 37,793 metastatic colorectal cancer patients: a populationbased, propensity score-adjusted trend analysis. Ann Surg 2015;262:112-20.

3. Venigalla S, Guttmann DM, Horne ZD, et al. Definitive local therapy is associated with improved overall survival in metastatic cervical cancer. Pract Radiat Oncol 2018;8:e377-85.

4. Ha GW, Kim JH, Lee MR. Meta-analysis of oncologic effect of primary tumor resection in patients with unresectable stage IV colorectal cancer in the era of modern systemic chemotherapy. Ann Surg Treat Res 2018;95:64-72.

5. Cauley CE, Block SD, Koritsanszky LA, et al. Surgeons' perspectives on avoiding nonbeneficial treatments in seriously ill older patients with surgical emergencies: a qualitative study. J Palliat Med 2016;19:529-37.

6. Miner TJ, Jaques DP, Karpeh MS, et al. Defining palliative surgery in patients receiving noncurative resections for gastric cancer. J Am Coll Surg 2004;198:1013-21.

7. Hofmann B, Håheim LL, Søreide JA. Ethics of palliative surgery in patients with cancer. Br J Surg 2005;92:802-9.

8. Wilcox AR, Wong SL. Trends in palliative surgical care. Adv Surg 2019;53:69-81.

9. Thomay AA, Jaques DP, Miner TJ. Surgical palliation: getting back to our roots. Surg Clin North Am 2009;89:27-41.

10. Miner TJ. Communication skills in palliative surgery: skill and effort are key. Surg Clin North Am 2011;91:355-66.

11. Klaristenfeld DD, Harrington DT, Miner TJ. Teaching palliative care and end-of-life issues: a core curriculum for 
surgical residents. Ann Surg Oncol 2007;14:1801-6.

12. Kim BJ, Aloia TA. Cost-effectiveness of palliative surgery versus nonsurgical procedures in gastrointestinal cancer patients. J Surg Oncol 2016;114:316-22.

13. Dunn GP. Surgery, palliative care, and the American College of Surgeons. Ann Palliat Med 2015;4:5-9.

14. Krouse RS, Nelson RA, Farrell BR, et al. Surgical palliation at a cancer center: incidence and outcomes. Arch Surg 2001;136:773-8.

15. Hall WJ. Lower extremity revascularization in nursing home residents: surgery as palliation. JAMA Intern Med 2015;175:957-8.

16. Freeman RK, Arevalo G, Ascioti AJ, et al. An assessment of the frequency of palliative procedures in thoracic surgery. J Surg Educ 2017;74:878-82.

17. Tseng WH, Yang X, Wang H, et al. Nomogram to predict risk of 30-day morbidity and mortality for patients with disseminated malignancy undergoing surgical intervention. Ann Surg 2011;254:333-8.

18. Cohen JT, Miner TJ. Patient selection in palliative surgery: Defining value. J Surg Oncol 2019;120:35-44.

19. Keung EZ, Liu X, Nuzhad A, et al. In-hospital and long-term outcomes after percutaneous endoscopic gastrostomy in patients with malignancy. J Am Coll Surg 2012;215:777-86.

20. Miner TJ, Jaques DP, Shriver CD. A prospective evaluation of patients undergoing surgery for the palliation of an advanced malignancy. Ann Surg Oncol 2002;9:696-703.

21. Hope JM, Pothuri B. The role of palliative surgery in gynecologic cancer cases. Oncologist 2013;18:73-9.

22. Paul Olson TJ, Pinkerton C, Brasel KJ, et al. Palliative surgery for malignant bowel obstruction from carcinomatosis: a systematic review. JAMA Surg 2014;149:383-92.

23. Miner TJ, Brennan MF, Jaques DP. A prospective, symptom related, outcomes analysis of 1022 palliative procedures for advanced cancer. Ann Surg 2004;240:719-26.

24. Maeda Y, Shinohara T, Katayama T, et al. A laparoscopic approach is associated with a decreased incidence of SSI in patients undergoing palliative surgery for malignant bowel obstruction. Int J Surg 2017;42:90-4.

25. Feuer DJ, Broadley KE, Shepherd JH, et al. Surgery for the resolution of symptoms in malignant bowel obstruction in advanced gynaecological and gastrointestinal cancer. Cochrane Database Syst Rev 2000;4:CD002764.

26. Dunn GP, Milch RA, Mosenthal AC, et al. Palliative care by the surgeon: how to do it. J Am Coll Surg
2002;194:509-37.

27. Miner TJ. Palliative surgery for advanced cancer: Lessons learned in patient selection and outcome assessment. Am J Clin Oncol 2005;28:411-4.

28. Miner TJ. The palliative triangle: Improved patient selection and outcomes associated with palliative operations. Arch Surg 2011;146:517.

29. Rao J, Tiruchelvarayan R, Lee L. Palliative surgery for cervical spine metastasis. Singapore Med J 2014;5 5:569-73.

30. Perone JA, Riall TS, Olino K. Palliative care for pancreatic and periampullary cancer. Surg Clin North Am 2016;96:1415-30.

31. Lilley EJ, Cooper Z, Schwarze ML, et al. Palliative care in surgery: defining the research priorities. Ann Surg 2018;267:66-72.

32. PelvEx Collaborative. Palliative pelvic exenteration: A systematic review of patient-centered outcomes. Eur J Surg Oncol 2019;45:1787-95.

33. Piper C, Porres D, Pfister D, et al. The role of palliative surgery in castration-resistant prostate cancer. Curr Opin Support Palliat Care 2014;8:250-7.

34. Moss AC, Morris E, Mac Mathuna P. Palliative biliary stents for obstructing pancreatic carcinoma. Cochrane Database Syst Rev 2006;(1):CD004200.

35. No JH, Kim SW, Lim C-H, et al. Long-term outcome of palliative therapy for gastric outlet obstruction caused by unresectable gastric cancer in patients with good performance status: endoscopic stenting versus surgery. Gastrointest Endosc 2013;78:55-62.

36. Nabozny MJ, Kruser JM, Steffens NM, et al. Patientreported limitations to surgical buy-in: A qualitative study of patients facing high-risk surgery. Ann Surg 2017;265:97-102.

37. Udelsman BV, Lilley EJ, Qadan M, et al. Deficits in the palliative care process measures in patients with advanced pancreatic cancer undergoing operative and invasive nonoperative palliative procedures. Ann Surg Oncol 2019;26:4204-12.

38. Schwarze ML, Zelenski A, Baggett ND, et al. Best Case/ Worst Case: ICU (COVID-19)-A Tool to Communicate with Families of Critically Ill Patients with COVID-19. Palliat Med Rep 2020;1:3-4.

39. Bakke KE, Miranda SP, Castillo-Angeles M, et al. Training surgeons and anesthesiologists to facilitate end-of-life conversations with patients and families: A systematic review of existing educational models. J Surg Educ 2018;75:702-21.

40. Baile WF, Buckman R, Lenzi R, et al. SPIKES-A six-step 
protocol for delivering bad news: application to the patient with cancer. Oncologist 2000;5:302-11.

41. Schwarze ML, Kehler JM, Campbell TC. Navigating high risk procedures with more than just a street map. J Palliat Med 2013;16:1169-71.

42. Kruser JM, Taylor LJ, Campbell TC, et al. "Best Case/Worst Case": Training surgeons to use a novel communication tool for high-risk acute surgical problems. J Pain Symptom Manage 2017;53:711-9.e5

43. Taylor LJ, Nabozny MJ, Steffens NM, et al. A framework to improve surgeon communication in high-stakes surgical decisions: Best Case/Worst Case. JAMA Surg 2017;152:531.

44. Berlin A, Kunac A, Mosenthal AC. Perioperative goalsetting consultations by surgical colleagues: a new model for supporting patients, families, and surgeons in shared decision making. Ann Palliat Med 2017;6:178-82.

45. Sun V, Krouse RS. Palliative surgery: incidence and outcomes. Semin Oncol Nurs 2014;30:234-41.

46. Wright AA, Zhang B, Ray A, et al. Associations between end-of-life discussions, patient mental health, medical care near death, and caregiver bereavement adjustment. JAMA 2008;300:1665-73.

47. Ballou JH, Brasel KJ. Palliative Care and Geriatric Surgery. Clin Geriatr Med 2019;35:35-44.

48. Lilley EJ, Gemunden SA, Kristo G, et al. Utility of the "surprise" question in predicting survival among older patients with acute surgical conditions. J Palliat Med 2017;20:420-3.

49. Alexander SC, Ladwig S, Norton SA, et al. Emotional distress and compassionate responses in palliative

Cite this article as: Louie AD, Miner TJ. Palliative surgery and the surgeon's role in the palliative care team: a review. Ann Palliat Med 2022;11(2):907-917. doi: 10.21037/apm-20-2381 care decision-making consultations. J Palliat Med 2014;17:579-84.

50. Robins B, Booser A, Lantos JD. When parents have misunderstandings about the risks and benefits of palliative surgery. Pediatrics 2018;142:e20180482.

51. Kee F, Owen T, Leathem R. Offering a prognosis in lung cancer: when is a team of experts an expert team? J Epidemiol Community Health 2007;61:308-13.

52. Hall DE, Arya S, Schmid KK, et al. Association of a frailty screening initiative with postoperative survival at 30, 180, and 365 Days. JAMA Surg 2017;152:233.

53. Lindvall C, Lilley EJ, Zupanc SN, et al. Natural language processing to assess end-of-life quality indicators in cancer patients receiving palliative surgery. J Palliat Med 2019;22:183-7.

54. Cooper Z, Courtwright A, Karlage A, et al. Pitfalls in communication that lead to nonbeneficial emergency surgery in elderly patients with serious illness: description of the problem and elements of a solution. Ann Surg 2014;260:949-57.

55. Miner TJ, Jaques DP, Tavaf-Motamen H, et al. Decision making on surgical palliation based on patient outcome data. Am J Surg 1999;177:150-4.

56. Funder JA, Jepsen KV, Stribolt K, et al. Palliative surgery for pseudomyxoma peritonei. Scand J Surg 2016;105:84-9.

57. Krouse RS. The International Conference on Malignant Bowel Obstruction: A Meeting of the Minds to Advance Palliative Care Research. J Pain Symptom Manage 2007;34:S1-6. 